\title{
Laparoscopy in the public health system in Ecuador: an evaluation of the status quo and the education in minimally invasive surgery
}

\author{
Harald Krentel • Mercedes Almagro Ruiz
}

Received: 1 November 2011 / Accepted: 1 December 2011 / Published online: 22 December 2011

(C) Springer-Verlag 2011

\begin{abstract}
The following study describes the use of laparoscopic surgery in the public hospitals of the Ecuadorian ministry of public health in Quito, Ecuador in the year 2008. The aim of the present study was the description of the recent situation of laparoscopic surgery in the public sector of the Ecuadorian capital, the level of training and education of laparoscopists, and the determination of missing factors in the educational programs in minimally invasive surgery in Ecuador. We analyzed the use of laparoscopic approach in correlation to the specialty and the diagnosis. The results revealed that in many diagnoses, the standard surgical approach still is the laparotomy. The discrepancy between general surgery, gynecology, and urology in the use of minimally invasive surgery in the public health system shows the different educational levels and the need of training programs at university level and in the hospitals especially in gynecology.
\end{abstract}

Keywords Gynecologic endoscopy · Training and education

H. Krentel
CEGPA, Centro de Endoscopía Ginecológica Peruano Aleman,
Lima, Peru

\section{H. Krentel $(\bowtie)$}

Städtisches Krankenhaus Eisenhüttenstadt,

Gynecology and Obstetrics,

Friedrich-Engels-Str. 39,

15890 Eisenhüttenstadt, Germany

e-mail: krentel@cegpa.org

M. A. Ruiz

Hospital Eugenio Espejo,

Quito, Ecuador

\section{Background}

Minimally invasive surgery has become the most important surgical approach in the last two decades in the surgical treatment of benign and malign intraabdominal diseases in general surgery, gynecology, and urology. Especially in gynecology in various centers of laparoscopy and hysteroscopy, more than $90 \%$ of the interventions are realized by endoscopic approaches. However there is a big discrepancy between the use of laparoscopy in different countries, especially in correlation with the status of general development. The success of laparoscopy in developed regions like North America and Europe is correlated with the demand of this minimally invasive procedure by the patients due to the well-known benefits - faster recuperation, less pain, and small incisions with a good cosmetic result. However only a well-informed patient can demand a new procedure and laparoscopic interventions can only be realized by trained surgeons. The present evaluation analyzes the educational level of Ecuadorian laparoscopists in the public sector, the use of laparoscopy in comparison with laparotomy, and the training situation in Ecuador.

\section{Methods and aim}

In a first step, we evaluated the daily practice and the personal experience of the surgeons with laparoscopy using a questionnaire. One hundred seven general surgeons, gynecologists, and urologists from the following hospitals were included to the study: Eugenio Espejo, Baca Ortiz, Isidro Ayora, Pablo Arturio Suarez, and Enrique Garces. In the second step, the database of the five public hospitals in Quito, Ecuador were analyzed comparing laparoscopy versus laparotomy in general surgery, gynecology, and urology. 
Finally we analyzed the postgraduate courses of all Ecuadorian universities with medical faculties. The aim of the study was to answer the following questions:

- What is the recent training level of laparoscopists working in the public hospitals in Quito, Ecuador?

- Do the surgeons participate in training workshops?

- Does an Ecuadorian certification system in laparoscopy exists?

- What is the status quo in laparoscopy in the public hospitals in Quito, Ecuador?

- What is the status quo in gynecologic laparoscopy in the public hospitals in Quito, Ecuador?

- Is there a quality standard in laparoscopy in Ecuador?

- How many Ecuadorian universities offer postgraduate programs in laparoscopy?

- Do the surgeons wish to continue their individual training?

\section{Results}

Evaluation of surgeons working in the public hospital of Quito, Ecuador

Fifty-eight out of 107 (54\%) physicians (surgeons, gynecologists, urologists) working at the public hospitals in the Ecuadorian capital responded to the questionnaire. Almost $90 \%$ of the interviewed surgeons already realize laparoscopies in their daily practice. However $44 \%$ of the laparoscopists perform a laparoscopic surgery only one to three times a month, while $40 \%$ answered to realize a laparoscopy one to three times a week and $16 \%$ only occasionally laparoscope their patients. All interviewed surgeons participated in one or more workshops of laparoscopic surgery in Ecuador or another country. The duration of the training in $69 \%$ of the cases was less than a month. Only $14 \%$ of the physicians commented to have participated a training program for more than 7 months. Half of the surgeons received a certification of participation (53\%), while $38 \%$ did not receive any certification after the training program. Only $9 \%$ of the realized laparoscopic educational programs were linked to an university including a respective certification. Before operating on humans, $76 \%$ of all interviewed surgeons exercised laparoscopy with animal models. However all physicians would like to continue their education in laparoscopy.

Evaluation of postgraduate courses at Ecuadorian universities

We analyzed the type of postgraduate courses the universities in Ecuador offer. The database of information in this case was the CONESUP (Consejo Nacional de Educación
Superior). This analyzation reveals that there is no postgraduate program at any of the eight Ecuadorian universities in laparoscopic surgery (Table 1).

Comparison of laparoscopy versus laparotomy in all realized surgeries in 2008

We analyzed all realized surgeries in the five public hospitals in Quito in the year 2008. Therefore the documentation of the operating theaters of these hospitals were investigated. We compared laparotomy versus laparoscopy in general surgery, urology, and gynecology. The approaches to treat the typical indications in the specialties were also compared. In 2008 a total of 7,963 surgical interventions has been realized in gynecology, urology, and general surgery in the five public hospitals of the Ecuadorian capital.

Almost $80 \%$ of all the interventions were conventional surgeries and only $20 \%$ minimally invasive surgeries. Comparing the results of the different hospitals the laparoscopic approach reached only $2 \%$ of more than 2,000 surgeries in the Hospital Baca Ortiz as a mínimum and 34.7\% as a maximum in the Hospital Isidro Ayora. In gynecology $90 \%$ of all interventions have been open surgeries. The most frequent gynecologic procedure was hysterectomy with 639 interventions. Only $13(2 \%)$ hysterectomies had been performed by laparoscopic approach in one of the institutions. In the same hospital, 22 of 45 surgeries for ovarian masses had been realized by laparoscopy (Table 2).

In the other 23 cases, a laparotomy was the choice to treat the ovarian tumor. Interestingly in case of infertility, the laparoscopy is the preferred approach in only $12.7 \%$ of the cases in three institutions. That means that $87.3 \%$ of the patients with infertility had been treated by a laparotomy.

Table 1 Universities with subspecialization in surgery (CONESUP)

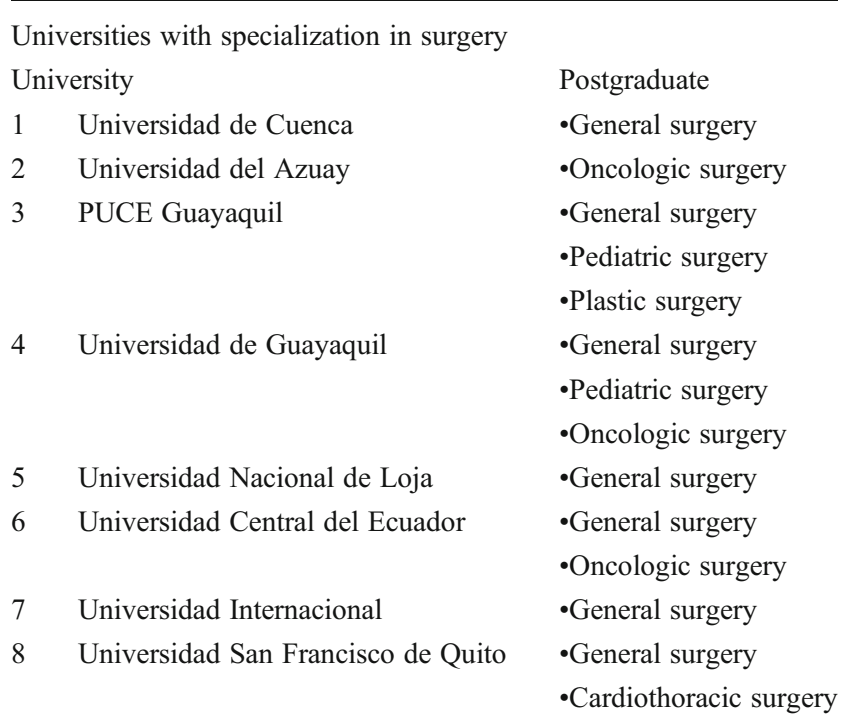


Table 2 Laparoscopic (LAP) versus conventional (CS) approaches in 2008

Public hospitals in Quito, Ecuador

\begin{tabular}{|c|c|c|c|c|c|c|c|c|c|c|c|c|}
\hline \multirow[t]{4}{*}{ Hospitals } & \multicolumn{12}{|c|}{ Specialties } \\
\hline & \multicolumn{2}{|c|}{ Surgery } & \multicolumn{2}{|c|}{ Urology } & \multicolumn{2}{|c|}{ Gynecology } & \multicolumn{4}{|c|}{ Subtotal } & \multirow[t]{3}{*}{ Total } & \multirow[t]{3}{*}{ Percentage } \\
\hline & \multirow[t]{2}{*}{ LAP } & \multirow[t]{2}{*}{$\mathrm{CS}$} & \multirow[t]{2}{*}{ LAP } & \multirow[t]{2}{*}{$\mathrm{CS}$} & \multirow[t]{2}{*}{ LAP } & \multirow[t]{2}{*}{$\mathrm{CS}$} & \multicolumn{2}{|l|}{ LAP } & \multicolumn{2}{|l|}{$\mathrm{CS}$} & & \\
\hline & & & & & & & No. & Percentage & No. & Percentage & & \\
\hline HEE & 325 & 1,114 & 4 & 163 & 15 & 136 & 344 & 19.57 & 1,413 & 80.42 & 1,757 & 100.00 \\
\hline HEG & 601 & 1,373 & 0 & 91 & 9 & 181 & 610 & 27.00 & 1,645 & 73.00 & 2,255 & 100.00 \\
\hline HOIA & 0 & 0 & 0 & 0 & 99 & 186 & 99 & 34.70 & 186 & 65.20 & 285 & 100.00 \\
\hline HBO & 16 & 953 & 5 & 35 & 21 & 988 & 42 & 2.08 & 1,976 & 97.92 & 2,018 & 100.00 \\
\hline HPAS & 501 & 668 & 20 & 31 & 30 & 398 & 551 & 33.43 & 1,097 & 66.50 & 1,648 & 100.00 \\
\hline Subtotal & 1,443 & 4,108 & 29 & 320 & 174 & 1,889 & 1,646 & 20.67 & 6,317 & 79.33 & 7,963 & 100.00 \\
\hline
\end{tabular}

For simple diagnostic reasons, the laparoscopy is the procedure of first choice. In $77.1 \%$ of the cases, the surgeons preferred the minimally invasive surgery for an intraabdominal diagnostic. In one hospital the percentage of laparoscopies reached almost $30 \%$ of the interventions. Due to the very rare endoscopic approach in the other hospitals, the total percentage of laparoscopies in the gynecologic departments of the public hospitals in Quito, Ecuador did not reach more than $10 \%$ of all surgeries (Table 3).

Interestingly in general surgery, the acceptance of laparoscopy seems to be bigger. After all $25 \%$ of 5,614 surgeries have been laparoscopies in the year 2008. The most important laparoscopic procedure is the cholecystectomy with $67 \%$ laparoscopic approach of all interventions, while the general surgeons in case of a diagnostic surgery prefer the open approach (98.9\%). Only $1.5 \%$ of 1,539 apendectomies had been performed by laparoscopy. However the Ecuadorian laparoscopy plays a more important role in general surgery compared with gynecology.

\section{Discussion}

Only $9.9 \%$ of all gynecologic surgeries in the public sector of the Ecuadorian capital are laparoscopies. That means that $90.1 \%$ of all gynecologic surgeries in the five most important public hospitals in the Ecuadorian capital were laparotomies in the year 2008. In gynecology of public hospitals in Ecuador, the laparoscopy still is just an approach in diagnostics and sterilization. As the results reveal, laparoscopy already is the standard procedure in cholecystectomy. Most of the general, gynegological, and urological surgeons working in public hospitals of the Ecuadorian capital Quito obtained basic skills in laparoscopy and participated in one or more workshops of minimally invasive surgery. Anyway their daily practice is not backed up by certifications.
In Ecuador, like in most of the other South American countries, a certification system in laparoscopy is not yet implemented. Neither the medical faculties of Ecuadorian universities nor the societies of gynecologic endoscopy in Ecuador offer a standardized education system or training programs in laparoscopic surgery respectively endoscopic gynecology. A certification of laparoscopic procedures for example by the Ecuadorian society of endoscopic gynecology would be a possible way to control the standards and the quality of the surgery. These certification systems are implemented since various years in Europe and North America. The German association of gynecologic endoscopy differs the individual education in three personal levels of laparoscopic and hysteroscopic procedures (MIC I-MIC III) and a standard for institutions to become a center of excellence in gynecologic endoscopy. Part of the certification process is the participation of the applicant in congresses and workshops of laparoscopy and hysteroscopy. The curriculum of the workshops is standardized by the respective working group [4]. These conditions lead to a controlled continuing education. However the most important part of the application procedure is the presentation of surgery reports. The required number of realized surgeries depends on the respective level. A minimum number of surgeries must be submitted to receive the first national level of endoscopic gynecology. The value of such a certification is undoubtable for the physician, particularly as insurance companies in a few years will demand on it. In the long term, the patients will look for certified surgeons considering the proved quality of their work. The common practice to offer laparoscopy without having the skills to perform it will be stopped by a transparent certification system.

As the results of this evaluation show, $100 \%$ of the interviewed surgeons trained themselves in animal models before operating on humans. Anyway $60 \%$ of the surgeons realize laparoscopies just occasionally or one to three times 


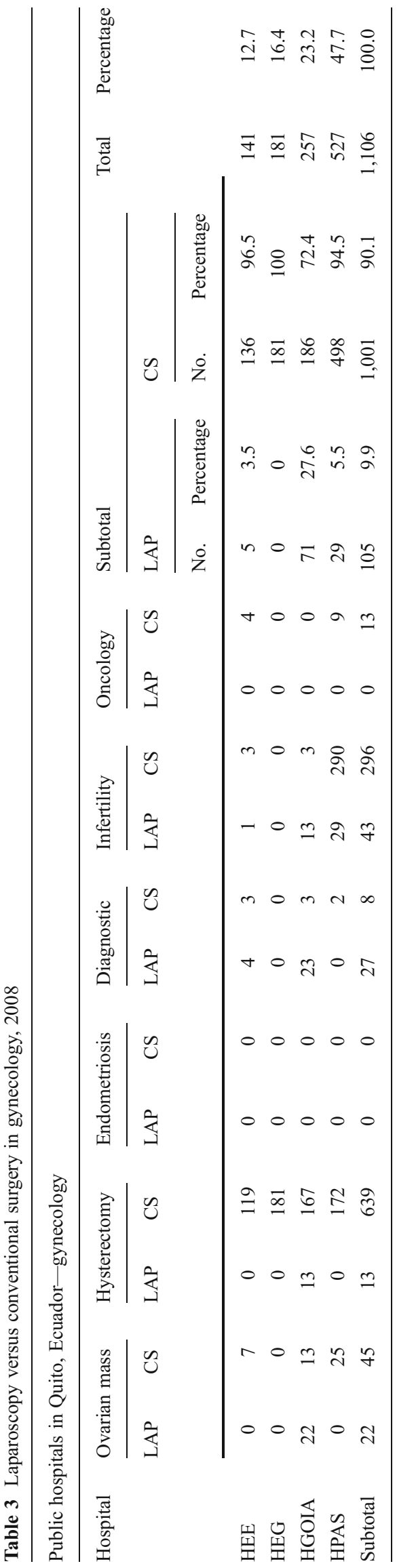

a month. The quality of laparoscopic surgery, the percentage of complications, and the duration particularly in more complex surgeries depend on the learning curve of the individual surgeons, which depends on the frequency of interventions. Morelli et al. publicated a comparison of vaginal versus laparoscopic hysterectomy, demonstrating the higher level of complications in laparoscopic hysterectomies only in the first year of the learning curve [13]. Boyd et al. showed that the rate of morbidity and mortality after hysterectomy depend on the number of performed procedures per year [3]. The percentage of persistent menstrual bleeding after subtotal laparoscopic hysterectomy is higher in surgeons with few experience [10]. In a prospective evaluation, Kirby et al. demonstrated the positive effect of training programs on the duration of a procedure [7]. Bojahr et al. analyzed 1,000 cases of subtotal laparoscopic hysterectomy, demonstrating an average time benefit of $13 \mathrm{~min}$ after a period of 4 years of performing the same procedure with a higher degree of difficulty [2]. The residents and young specialists must given the chance to assist laparoscopic procedures as often as possible under the supervision of a qualified laparoscopist. This could be part of the curriculum of a postgraduate university level education. The interest of all interviewed surgeons to continue their education is very high. Unfortunately the doors of the operating theaters of the private gynecologic institutions with high percentages and wider indications in gynecologic endoscopy are often closed to most of the residents. This is for legal reasons and also for economic reasons.

To educate means to open a lucrative market to competitors. An Australian publication revealed the lack of instruments and materials, training, experience, and the encouragement by colleagues as the main reasons to not perform more complex laparoscopic surgeries like myomectomies or hysterectomies [6]. Marret et al. concluded that skilled surgeons in laparoscopy and in laparoscopic myomectomy must help their trainees during their learning curve in order to decrease laparoconversions [12], respectively, in order to allow them the safe performance of more complex surgeries. In 2007 Kolkman et al. [8] published the current status of the implementation of operative laparoscopy in gynecology in the Netherlands, based on a questionnaire which had been sent to all hospitals $(n=102)$ in 2003. The total number and type of laparoscopic procedures performed in 2002 stratified by level of difficulty was compared to the results from 1993. The diffusion and acceptance of level 2 procedures (adhesiolysis, ectopic pregnancy, laparoscopic treatment of endometriosis, cystectomy, oophorectomy, LAVH) increased significantly. Anyway the diffusion of LAVH was only $58 \%$. The authors concluded that the implementation of operative gynecological laparoscopy in the Netherlands seems to develop at a slow pace. A Canadian survey from 2007 revealed that $24.5 \%$ of Canadian 
gynecologists performed laparoscopic myomectomy. Of the respondents $70.7 \%$ felt that lack of training was the main barrier to perform complex laparoscopic procedures [11]. Another recent Canadian study [9] revealed that Canadian residents in obstetrics and gynecology are not receiving adequate training to feel comfortable using the laparoscopic approach as opposed to the vaginal and abdominal routes. The positive effects of training and education in laparoscopy has been shown in various publications. Edelman et al. for example [5] described the impact of the integration of laparoscopic skill training in the medical school curriculum on the final surgical performance of residents. A French prospective study revealed that simulator training provides a more rapid acquisition of competence in surgical technique [1]. Morgan Ortiz et al. described the teaching of total laparoscopic hysterectomy in a Mexican university training program in gynecologic laparoscopy. Eighteen participants received the respective university diploma in the last 2 years [14]. Further national and international educational initiatives are needed to ensure that graduates and specialists are capable of performing more different and complex types of endoscopic surgery in gynecology, but also urology and general surgery.

\section{Conclusion}

In the public Ecuadorian health sector, laparoscopy still does not play an important role in general surgery, urology, and particularly in gynecology. The lack of training programs and a frequent access to more complex interventions under supervision of a skilled specialist does not allow the motivated surgeons to develop their skills in laparoscopy. A national concept of resident and specialist training is needed to implement laparoscopy as a frequent procedure in a wider range of indications and to make it a safe and cost-effective alternative to conventional surgery for the population.

Declaration of interest The authors report no conflicts of interest. The authors alone are responsible for the content and writing of the paper.

\section{References}

1. Beyer L, Troyer JD, Mancini J, Bladou F, Berdah SV, Karsenty G (2011) Impact of laparoscopy simulator training on the technical skills of future surgeons in the operating room: a prospective study. Am J Surg 202(3):265-272

2. Bojahr B, Tchartchian G, Ohlinger R (2009) Laparoscopic supracervical hysterectomy: a retrospective analysis of 1000 cases. JSLS 13(2):129-134

3. Boyd LR, Novetsky AP, Curtin JP (2010) Effect of surgical volumen on the route of hysterectomy and short-term morbidity. Obstet Gynecol 116(4):909-915

4. DeWilde RL, Hucke J, Kolmorgen K, Tinneberg H (2011) Recommendations by the Gynecologic Endoscopy Working Group of the German Society of Obstetrics and Gynecology for the advancement of training and education in minimal-access surgery. Arch Gynecol Obstet 283(3):509-512

5. Edelman DA, Mattos MA, Bouwman DL (2011) Impact of fundamentals of laparoscopic surgery training during medical school on performance by first year surgical residents. J Surg Res 170(1):6-9

6. Englund M, Robson S (2007) Why has the acceptance of laparoscopic hysterectomy been slow? Results of an anonymous survey of Australian gynecologists. J Minim Invasive Gynecol 14(6):724-728

7. Kirby TO, Numnum TM, Kilgore LC, Straughn JM (2008) A prospective evaluation of a simulator-based laparoscopic training program for gynecology residents. J Am Coll Surg 206(2):343-348

8. Kolkman W, Trimbos-Kemper TC, Jansen FW (2007) Operative laparoscopy in the Netherlands: diffusion and acceptance. Eur J Obstet Gynecol Reprod Biol 130(2):245-248

9. Kroft J, Moody J, Lee P (2011) Canadian hysterectomy educational experience: survey of recent graduates in obstetrics and gynecology. J Minim Invasive Gynecol 18(4):438-444

10. Lieng M, Qvigstad E, Istre O, Langebrekke A, Ballard K (2008) Long-term outcomes following laparoscopic supracervical hysterectomy. BJOG 115(13):1605-1610

11. Liu G, Zolis L, Kung R, Melchior M, Singh S, Cook EF (2010) The laparoscopic myomectomy: a survey of Canadian gynaecologists. J Obstet Gynaecol Can 32(2):139-148

12. Marrett H, Chevillot M, Giraudeau B, Study Group of the French Society of Gynaecology and Obstetrics (Quest Division) (2006) Factors influencing laparoconversions during the learning curve of laparoscopic myomectomy. Acta Obstet Gynecol Scand 85 (3):324-329

13. Morelli M, Caruso M, Noia R, Chiodo D, Cosco C, Lucia E, Biamonte M, Zullo F (2007) Total laparoscopic hysterectomy versus vaginal hysterectomy: a prospective randomized trial. Minerva Ginecol 59(2):99-105

14. Morgan Ortiz F, Lopez Zepeda MA (2011) Teaching of total laparoscopic hysterectomy in a gynecological laparoscopy university diploma course. Ginecol Obstet Mex 79(9):547-552 